\title{
Kinetics and enthalpy measurements of interaction between $\beta$-amyloid and liposomes by surface plasmon resonance and isothermal titration microcalorimetry
}

\author{
Ming-Shen Lin ${ }^{a}$, Hsiu-Mei Chiu ${ }^{a}$, Fu-Jung Fan ${ }^{\text {a }}$, Hui-Ting Tsai ${ }^{\text {a }}$, \\ Steven S.-S. Wang ${ }^{\mathrm{b}}$, Yung Chang ${ }^{\mathrm{c}}$, Wen-Yih Chen ${ }^{\mathrm{a}, *}$ \\ a Department of Chemical and Materials Engineering, National Central University, Jhong-Li 320, Taiwan \\ ${ }^{\mathrm{b}}$ Department of Chemical Engineering, National Taiwan University, Taipei 10617, Taiwan \\ ${ }^{\mathrm{c}} R \& D$ Center for Membrane Technology and Department of Chemical Engineering, Chung Yuan Christian University, Jhong-Li, 320, Taiwan
}

Received 12 November 2006; received in revised form 13 March 2007; accepted 20 March 2007

Available online 27 March 2007

\begin{abstract}
The objective of this research is to understand the interaction mechanism of $\beta$-amyloid (A $\beta$ ) with cell and were basically divided into two parts. The first part focused on the time-dependent structural changes of A $\beta$ (1-40) by circular dichroism (CD) spectroscopy, thioflavin T (ThT) fluorescence assay, and atomic force microscopy (AFM). The second part emphasized the kinetics and enthalpy of interaction between A $\beta$ (1-40) and liposome by surface plasmon resonance (SPR) and isothermal titration microcalorimetry (ITC). Results obtained from CD, ThT and AFM confirmed the formation of $1 \mu \mathrm{m}$ fibril after single day incubation. The driving force of kinetic interaction between A $\beta$ and liposomes was revealed by SPR to be electrostatics. Further studies indicated that fresh A $\beta$ has high GM1 affinity. Besides, addition of cholesterol to the liposome could alter membrane fluidity and affect the interactions of fresh $A \beta$ with liposomes especially in the amount of $A \beta$ absorbed and preserving the structure of liposome after adsorbing. Hydrophobicity was found to be the driving force leading to the interaction between A $\beta$ fibrils and liposomes. These reactions are endothermic as supported by ITC measurements. When the composition of liposomes is zwitterionic lipids, the interaction of A $\beta$ with liposomes is predominantly hydrophobic force. In contrast, the driving force of interaction of charged lipids with $A \beta$ is electrostatic.
\end{abstract}

(C) 2007 Elsevier B.V. All rights reserved.

Keywords: $\beta$-Amyloid (A $\beta$ ); Lipsome; Surface plasmon resonance; Isothermal titration calorimetry; Enthalpy

\section{Introduction}

To date, over 20 peptides or proteins have been identified to form amyloid fibrils or aggregates via abnormal folding in the body and are thought to cause diseases named as protein folding disorders (PFDs) [1] or protein conformational diseases (PCDs) [2]. These diseases include Alzheimer disease (AD), Parkinson disease (PD), type II diabetes, and bovine spongiform encephalopathy (BSE) associated with $\beta$-amyloid (A $\beta$ ), $\alpha$-synuclein, human amylin, and prion protein, respectively [3]. However, the mechanism by which amyloid fibrils cause cytotoxicity and disease is still not clear.

\footnotetext{
* Corresponding author. Tel.: +886 34227151x34222; fax: +88634225258. E-mail address: wychen@cc.ncu.edu.tw (W.-Y. Chen).
}

$\mathrm{AD}$ is the most common neurodegenerative disease occurred in elders characterized pathologically by the presence of senile plaques and neurofibrillary tangles in brain [4]. Senile plaques have been of great interest to many researchers due to its implication in $\mathrm{AD}$. Additionally, according to previous researches, $A \beta$, the main constituents of senile plagues, is a self-assemble amphiphilic peptide with the distinct character that is toxic-free in its monomer form and becomes cytotoxic during its transition to fibril form [5-6]. As of now, the mechanisms at which $A \beta$ leads to the death of cells are still unclear.

Subsequently, multiple researchers have devoted their studies on the interaction of $\mathrm{A} \beta$ monomers and the absorption mechanism of $A \beta$ on the cell membrane. Many references have reported factors in influencing the structural changes of $A \beta$, such as incubation temperature [6,7], the choice of solvents [8], the $\mathrm{pH}$ of buffer [9], concentration and length of $\mathrm{A} \beta$ species, the inclusion of nuclei and liposome and various compositions of 
liposome [10-19]. With reference to the papers cited above, we concluded that structural change of $A \beta$ could easily be affected by these factors and the presence of liposome accelerated the transition from $A \beta$ monomer to fibril.

The state at which the $A \beta$ is most likely to interact with cell membrane is an important issue. The formation of aggregates on the cell membrane, whether it be initiated by monomeric $A \beta$ or aggregated $A \beta$, remains unknown. With the aid of surface plasmon resonance (SPR) and isothermal titration microcalorimetry (ITC), we obtained valuable kinetic and thermodynamic information to show the interaction mechanisms between $A \beta$ of various states and liposomes of various compositions.

\section{Materials and methods}

\subsection{Materials}

$\mathrm{A} \beta$ (1-40) was purchased from Biosource, dipalmitoyl phosphatidyl glycerol (DPPG) and cholesterol were purchased from Sigma, L- $\alpha$-dipalmitoyl phosphatidylcholine (DPPC) was purchased from Acros, ganglioside GM1 was purchased from Avanti Polar Lipids, 1,1,1,3,3,3-hexafluoro-2-propanol (HFIP) was purchased from Aldrich. Thioflavin $\mathrm{T}$ was purchased from Fluka.

\subsection{A $\beta$ sample preparation [9]}

$\mathrm{A} \beta$ stock solution was prepared by dissolving the peptide at $1 \mathrm{mg} / \mathrm{ml}$ in HFIP to render $A \beta$ monomeric. The required sample of stock solution was transferred to the tube and the volatile solvent was removed using a vacuum system over-night, then the lyophilized amorphous powders of $\mathrm{A} \beta$ was dissolved in PBS (10 mM Na $2 \mathrm{HPO}_{4} / \mathrm{KH}_{2} \mathrm{PO}_{4}, 100 \mathrm{mM} \mathrm{NaF}, \mathrm{pH}$ 7.4) and allowed to incubate at room temperature. In order to avoid the influence of HFIP on SPR signal, HFIP was removed from the stock solution by evaporation rather than dilution into PBS.

\subsection{Liposome preparation}

Lipid dissolved in chloroform/methanol (2:1, v/v) was placed in thermostat flask at $50^{\circ} \mathrm{C}$. The organic solvent was evaporated by nitrogen and a homogenous thin film was formed at the inner wall of the flask. Lipid films were hydrated with PBS by ultrasonic homogenization at $50{ }^{\circ} \mathrm{C}$ for $20 \mathrm{~min}$. The resulting colloidal suspension of multilamellar vesicles (MLVs) was subjected to three cycles of freezing in liquid nitrogen and thawing in warm water $\left(50^{\circ} \mathrm{C}\right)$. The small unilamellar vesicles (SUVs) were prepared from the MLV dispersions using an ultrasonic homogenizer (Sonopuls HD 200, Bandelin, England) in power MS 72/D, cycle 10 for $30 \mathrm{~min}$ and extruded with a cellulose acetate membrane (pore size $=0.22 \mu \mathrm{m}$ ) to allow the homogenous distribution of the vesicle size.

\subsection{Modification of sensor chip [20]}

The gold surface of chip was first cleaned by washing with absolute alcohol. The fresh chips were submerged into the
$1 \mathrm{mM}$ (MHA) alcohol solution for $24 \mathrm{~h}$ to form a carboxyl selfassembled monolayer (SAM) on the gold surface. The chips were then transferred into an anhydrated (DMF) solution of $0.05 \mathrm{M}$ TFAA and $0.1 \mathrm{M}$ (TEA) for $20 \mathrm{~min}$ after rinsing with absolute alcohol. The chips were merged into the $28 \mathrm{mM}$ Brij76 solution for $30 \mathrm{~min}$ following rinsing with dichloromethane. Finally, the sensor chips with Brij-76 derivatized surface for immobilizing the lipid bilayer were obtained after rinsing with absolute alcohol and drying with nitrogen.

\subsection{Circular dichroism (CD) spectroscopy [14]}

CD measurements were carried out using a Jasco J-810 spectropolarimeter. All measurements were performed at room temperature. The path length of quartz cell was $1 \mathrm{~mm}$. All spectra were corrected by subtracting the buffer baseline. CD spectra in the far UV range (190-250 nm) were obtained.

\subsection{Thioflavin T(ThT) fluorescence spectroscopy}

Fluorescence measurements were carried out on a Jasco FP-6500. A $\beta$ fibril formation was assessed using the method described by LeVine [21]. Thioflavin T was dissolved in PBS. The Thioflavin $T$ solution and $A \beta$ sample was mixed at 1:1 molar ratio and the final concentration of $A \beta$ is $20 \mu \mathrm{M}$. Fluorescence emission spectra were taken from 350 to $650 \mathrm{~nm}$ at an excitation wavelength of $440 \mathrm{~nm}$ and emission maximum at $485 \mathrm{~nm}$ was indicative of the presence of amyloid fibrils.

\subsection{Atomic force microscopy (AFM)}

The surface of wafer was cleaned by sequential washing with acetone ( $2 \mathrm{~min})$, isopropanol $(2 \mathrm{~min})$ and di-water $(2 \mathrm{~min})$ in the ultrasonic device and consequently dried using nitrogen. The fresh wafer was soaked in $20 \mu \mathrm{M} A \beta$ solution for $30 \mathrm{~min}$. A $\beta$ coated surfaces were dried with nitrogen before the images were taken.

\subsection{Surface plasmon resonance (SPR)}

This experiments were performed as our previously study [22]. A homemade angle resolved SPR in the Kretschmann configuration was used to attenuate total refection (ATR), combined with the flow analysis system [23]. Sensor chip after modification was soaked in liposome solution for $24 \mathrm{~h}$ to immobilize liposomes onto the surface. The morphology of chip with liposomes was analyzed by AFM before and after interacting with protein. The liposomes were chemically immobilized on the sensor chip, and PBS was introduced into the sensor flow cell at $25 \mu \mathrm{l} / \mathrm{min}$. As the SPR resonance angle reached a plateau, the equilibrium RU value was defined as baseline. Then the introduction of $20 \mu \mathrm{M} \mathrm{A} \beta$ solution resulted in resonance angle shifted with respect to time, meaning the refractive index of interface was altered when $A \beta$ adsorbed on the liposome membrane on the chip surface. After the signals were stabilized as the adsorption reach balance, the PBS buffer was pumped into the sensor cell to elute or dissociate (the arrow point in the figures) 
the weakly or nonspecifically bounded $\mathrm{A} \beta$. All experiments were performed at $25^{\circ} \mathrm{C}$.

\subsection{Isothermal titration microcalorimetry (ITC)}

The reaction enthalpy measurements were performed using a Thermal Activity Monitor (Thermometric AB, Sweden) controlled by Digitam software. The microreaction system was carried out in a titration mode with a $4 \mathrm{ml}$ stainless steel ampoule. When thermal equilibrium between the ampoule and the heat sink was reached, a $20 \mu \mathrm{l}$ aliquot of $75 \mu \mathrm{M}$ fresh $\mathrm{A} \beta$ solution was injected for 12 times at a constant interval of $30 \mathrm{~min}$ via a $250-\mu l$ rotating stirrer-syringe, into the reaction cell (ampoule) containing $109 \mu \mathrm{M}, 2 \mathrm{ml}$ liposome in $10 \mathrm{mM}$ PBS. The titration experiments were carried out using peptide/lipid in the molar ratio of $0.007-0.083$ and at a constant temperature $\left(25^{\circ} \mathrm{C}\right)$. In control experiments, the fresh $A \beta$ solutions were titrated into pure buffer to obtain the dilution heat, which was subtracted from the experimental curve in the final analysis.

\section{Results and discussion}

\subsection{A $\beta$ structure}

Self-aggregating properties of $A \beta$ peptides were analyzed using three different techniques: circular dichroism spectroscopy, ThT fluorescence and AFM.

\subsubsection{Circular dichroism spectroscopy}

Conformational changes of peptides and proteins in solution were studied by CD. A minimum peak at $197 \mathrm{~nm}$ seen from Fig. 1 was a characteristic signature of random coils [6]. As the incubation time increases, the characteristic peak shifted from $197 \mathrm{~nm}$ to 205-220, signifying the formation of an ordered structure. In addition, a structural transition with an isodichroic point at about $200 \mathrm{~nm}$ provides evidence that simple two-state equilibrium (random coil $\leftrightarrow \beta$-sheet) has occured for $\mathrm{A} \beta(1-40)$ at $20 \mu \mathrm{M}[15]$.

\subsubsection{Thioflavin T fluorescence assay}

ThT, a type of hydrophobic fluoresce probes, can easily differentiate the degree of $\mathrm{A} \beta$ fibril formation. Subsequently, a thorough analysis of the correlation between time and the extent of fibril formation was the main objective of this research. As shown in Fig. 2, a dramatic increase in the ThT fluorescence

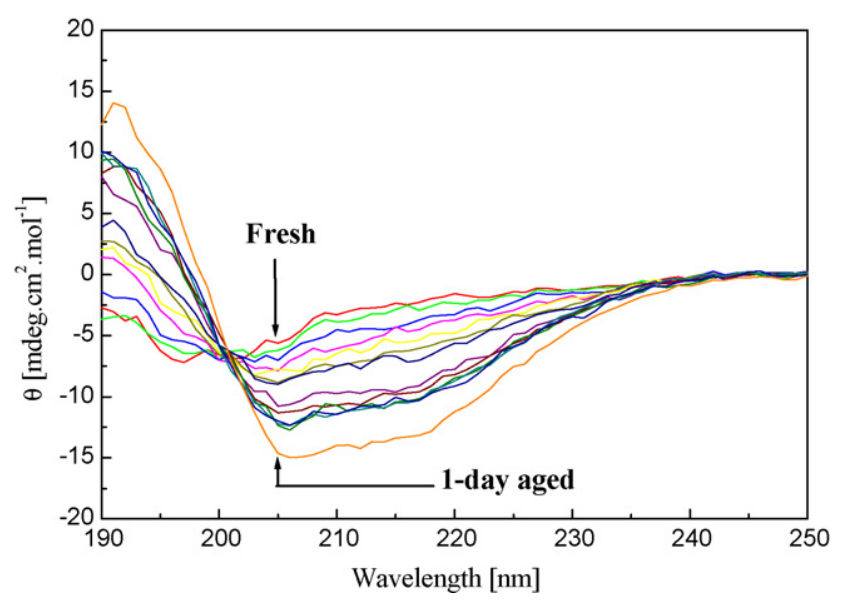

Fig. 1. Circular dichroism spectra of time-course of $20 \mu \mathrm{M} \mathrm{A} \beta(1-40)$ at $25^{\circ} \mathrm{C}$.

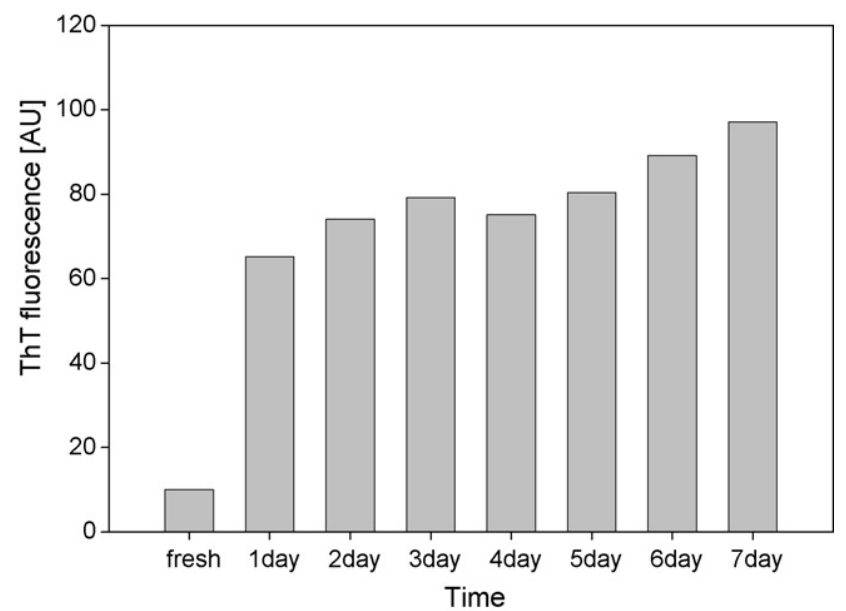

Fig. 2. Time-course of fibril formation of $20 \mu \mathrm{M} \mathrm{A} \beta(1-40)$ at $25^{\circ} \mathrm{C}$ using $\mathrm{ThT}$ fluorescence.

intensity was observed within 1-day incubation of $A \beta$. However, no substantial difference in the fluorescence intensity was found following 2-7 days of incubation. Results from ThT fluorescence revealed that $A \beta$ fibril (1-40), in large quantity, was formed after incubation for 1 day.

\subsubsection{AFM images}

From $A F M$ images, it was found that $A \beta$ began aggregating and became bulky particles after 1-h of incubation (Fig. 3A).
(A)

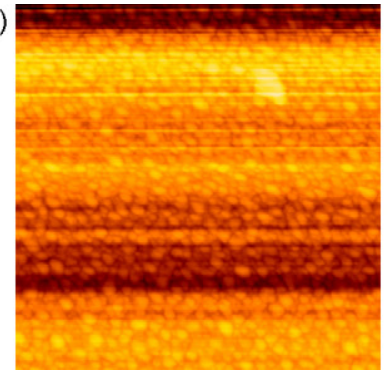

(B)

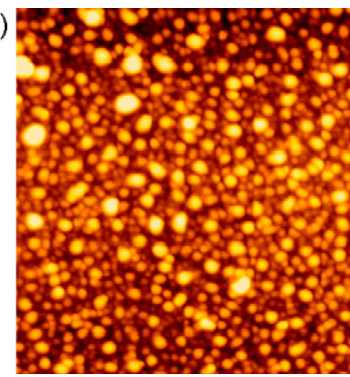

(C)

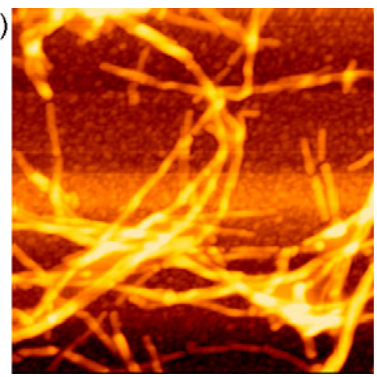

(D)

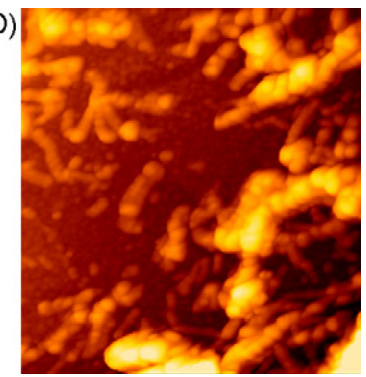

Fig. 3. Time-course of $20 \mu \mathrm{M} \mathrm{A \beta}(1-40)$ at $25^{\circ} \mathrm{C}$ using AFM. A-D are incubation for $1 \mathrm{~h}, 2 \mathrm{~h} ., 1$ day and 7 days, respectively. 
Within an hour, these bulky particles, though only a few in numbers would eventually increase in quantity over time. Incubating for $2 \mathrm{~h}$, the aggregation, as predicted, became more obvious (Fig. 3B). In addition, the formation of fibrils was detectable after 1 day of incubation (Fig. 3C). These fibrils, though may vary in their length, became more sturdy as observed under AFM after a week of incubation (Fig. 3D).

The morphology of $A \beta$ aggregation was observed from the AFM images. When $A \beta$ was incubated for 1 day, $A \beta$ fibrils were formed. The results as reported by Kremer et al. [19], using the electron microscope and bis-ANS fluorescence assay, indicated that the formation of $\mathrm{A} \beta$ fibrils at $\mathrm{pH} 6.0$ was driven by electrostatic force. However, the hydrophobic interaction was the driving force at $\mathrm{pH} 7.0$ and is similar to our AFM image as observed after of $A \beta$ was incubated after 1 day. With reference to other researches, our work demonstrated that the mechanism of $A \beta$ aggregation under this experimental condition is hydrophobic force in nature.

\subsection{A $\beta$-liposome interactions}

Results from ThT fluorescence assay and images of AFM confirmed that $20 \mu \mathrm{M} \mathrm{A} \beta(1-40)$ at $25^{\circ} \mathrm{C}$ had a dramatic change in structure after incubation for 1 day. Therefore, fresh and 1day incubated $A \beta$ species were used to interact with liposome for the following studies.

\subsubsection{Kinetics}

Sensorgram of fresh $20 \mu \mathrm{m} \mathrm{A} \beta$ (1-40) interacting with liposomes with various compositions is shown in Fig. 4. A diverse liposome samples were prepared as follows: DPPC, DPPG, DPPG with $20 \%$ cholesterol, a mixture of DPPC and DPPG in the ratio of 3-1, and a mixture of $5 \mathrm{~mol}$ of DPPC, 3 mol of GM1 and $2 \mathrm{~mol}$ of cholesterol. From Fig. 4, the ascending slopes of all five lines show adsorption while dissociation occurred when the slopes became negative after passing the maximum point. The adsorption and dissociation behaviors of these five different liposomes are plotted in Fig. 4. DPPG and DPPC had the highest

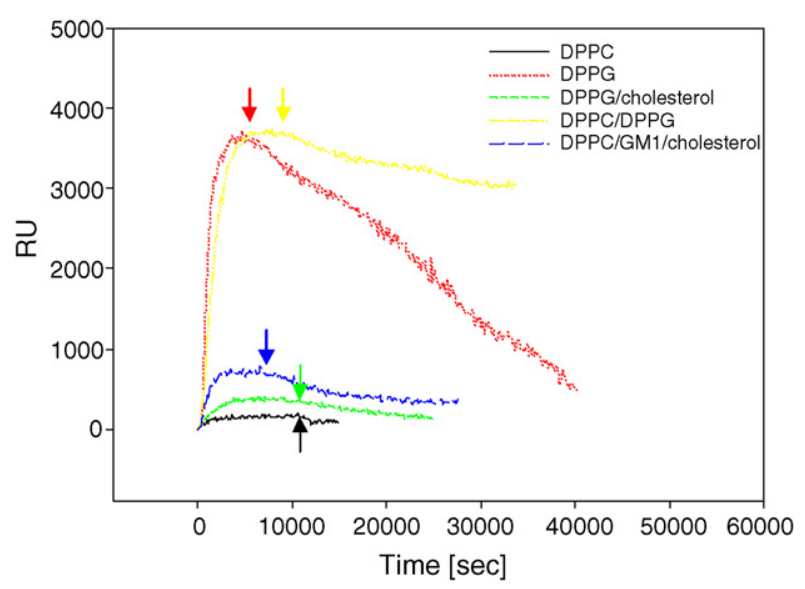

Fig. 4. The sensorgram of interactions of fresh $20 \mu \mathrm{M} \mathrm{A} \beta$ (1-40) with various liposome by SPR. The arrow indicates the initial position of buffer wash. and lowest initial rates of adsorption, respectively. In the dissociation phase, various liposomes showed different dissociation behavior. The physiological factor which contributed to their different adsorbing behavior was the lipid head group-DPPC, a zwitterionic lipid. Likewise, DPPG also with zwitterionic characteristics has a negative-charged head. GM1, meanwhile, has a salic acid residue that is negatively charged. Our data demonstrated that the lipid head group plays a role in the interactions between $A \beta$ and liposomes.

The dramatic increase of RU values of DPPG and DPPC/DPPG during the adsorption phase suggested that either the vesicles had ruptured without being stabilized by cholesterol or $\mathrm{A} \beta$ in higher quantity had adsorbed on the lipid vesicles. Also, it should be noted that during the dissociation phase, the RU value of DPPG monotonically decayed and eventually fell below the initial baseline. This behavior provided another proof that liposome immobilized on the sensor chip had either broken apart or had it's conformation changed. Results obtained from AFM confirmed that the structure of liposome had been altered into lipid bilayer from vesicle as a consequence of $A \beta$ interaction (data not shown).

In contrast with DPPG, the signals of liposomes consisting of DPPG with $20 \%$ cholesterol were found to be smaller than 1000 RU units. During the association phase, DPPG with $20 \%$ cholesterol has lower values of both initial rate of adsorption and adsorbed amount of $A \beta$ than those of DPPG. Due to the presence of cholesterol within the membrane, a reduction in the membrane fluidity was observed. This affected the amount of $A \beta$ adsorbed onto the liposome. However, unlike DPPG, the RU signal of DPPG with $20 \%$ cholesterol did not decrease monotonically in the dissociation phase, implying that the liposome stability was enhanced and membrane disruption was not encountered upon the addition of cholesterol. As for DPPC/GM1/cholesterol combination, a faster initial rate of adsorption and larger amount of adsorbed $\mathrm{A} \beta$ were observed relative to DPPG/cholesterol. Although both GM1 and DPPG have negatively charged head groups, the mechanisms of $A \beta$ binding to liposome were different. In addition, both the presence of GM1 and DPPG in the vesicles exhibited an irreversible nature in the dissociation phase, implying that the adsorbed $\mathrm{A} \beta$ on the surface of liposomes could not be removed by washing with PBS. It suggests that the absorbed $A \beta$ may go through structural conversion and aggregation and lead to some degree of irreversible adsorption. The interaction between DPPC liposome and $\mathrm{A} \beta$ had the smallest initial rate of adsorption, the faster initial rate of desorption, and the smallest amount of adsorbed $A \beta$, suggesting that a relatively weak interaction occurred between $A \beta$ and DPPC. Affinity constants involved in the interaction of $A \beta$ with various liposomes were determined by Langmuir binding model [24] and the results are reported in Table 1.

The relationship in the order of binding strength between fresh $\mathrm{A} \beta$ and liposomes with various compositions can be represented as follows: DPPG $>$ DPPC/DPPG (3:1 molar ratio) $>$ DPPC/GM1/cholesterol (5:3:2 molar ratio) $>$ DPPG/ $20 \%$ cholesterol $>$ DPPC (see Table 1). DPPG liposome has the highest affinity constant of $10^{7} \mathrm{M}^{-1}$ while DPPC liposome has 
Table 1

Affinities for fresh/incubation 1 day $A \beta$ (1-40) binding to various liposome

\begin{tabular}{llc}
\hline The state of A $\beta$ & Various liposome & $K_{\mathrm{A}}\left(\times 10^{5} \mathrm{M}^{-1}\right)$ \\
\hline & DPPC & 1.5 \\
& DPPG & 130 \\
Fresh & DPPG 20 mol\% cholesterol & 25 \\
& DPPC/DPPG (3:1 molar ratio) & 37 \\
& DPPC/GM1/cholesterol (5:3:2 & 12 \\
& molar ratio) & \\
& DPPC & 25 \\
Incubation & DPPG & 2.8 \\
1 & DPPG 20 mol\% cholesterol & 21 \\
day & DPPC/DPPG (3:1 molar ratio) & 17 \\
& DPPC/GM1/cholesterol (5:3:2 & 4.5 \\
& molar ratio) & \\
\hline
\end{tabular}

the smallest $\left(10^{5} \mathrm{M}^{-1}\right)$. Our affinity strength results suggested that the fresh $\mathrm{A} \beta$-liposome interaction is electrostatic-driven. Other researchers have reported that $A \beta$ has high affinity for the negatively charged lipids relative to the zwitterionic lipids [16]. Our results are in agreement with this view.

The GM1 liposome has high affinity than DPPG, indicating that GM1 was specifically recognized by $A \beta$. Other researchers pointed out that in human neuroblastoma cells, approximately half of all detergent-insoluble $\mathrm{A} \beta$ was present in the lipid rafts-rich ganglioside, sphingomyelin (SM), and cholesterol [2]. Ariga et al. have reported that neutral glycoshingolipid had a lower affinity for $\mathrm{A} \beta$ (1-40) than gangliosides [18]. These data seemed to indicate that the gangliosides are important for the binding between $A \beta$ and liposomes.

Fig. 5 shows the SPR sensorgrams of $20 \mu \mathrm{M} A \beta(1-40)$ after incubation for 1 day with various compositions of liposomes. Our results showed that the lipid head group had an impact on the binding behavior between $A \beta$ 1-day incubation and liposome. In the sensorgrams (Fig. 5), arrows are used to point out the start of washing-off by PBS in the dissociation phase. It can be found that the irreversible phenomenon was seen in all experiments. The binding strength

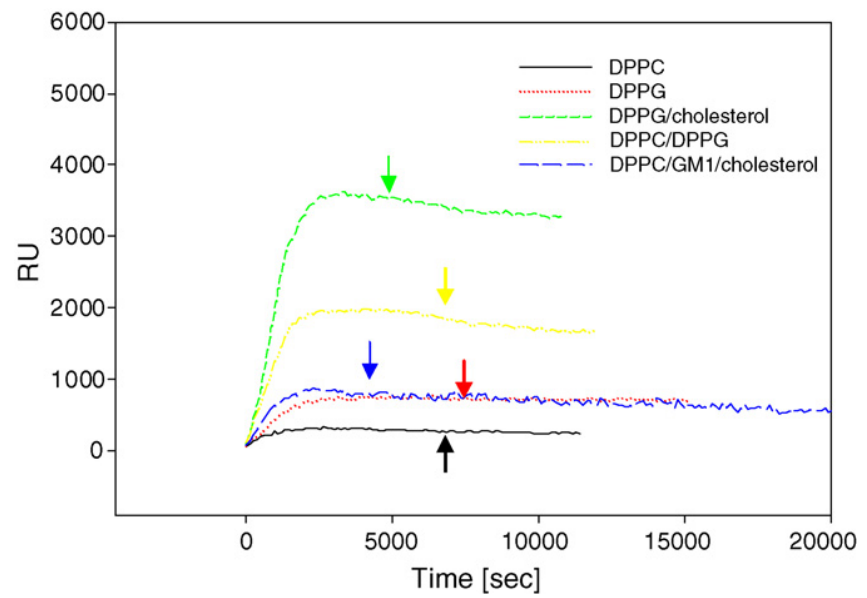

Fig. 5. The sensorgram of interactions of incubation 1 day $20 \mu \mathrm{M} A \beta(1-40)$ with various liposome by SPR. The arrow indicates the initial position of buffer wash. between liposomes and $\mathrm{A} \beta$ with 1-day incubation followed the order: DPPC $>$ DPPG/20\% cholesterol $>$ DPPC/DPPG $(3: 1$ molar ratio) $>$ DPPG $>$ DPPC/GM1/cholesterol (5:3:2 molar ratio) (see Table 1). It is suggested from the affinity strength data that the binding between 1-day incubation $A \beta$ and various liposomes is attributed to the hydrophobic force.

Our data showed that both fresh and 1-day incubation $A \beta$ were able to interact with liposomes but may be following different mechanisms. As shown in Table 1, the binding affinities of the aged (1-day incubation) $A \beta$ with the immobilized liposome are generally lower than that of the fresh A $\beta$, except for DPPC, indicating that there is different binding mechanism between $\mathrm{A} \beta$ with liposome for different states of $A \beta$. Furthermore, for incubated $A \beta$, most of the $A \beta$ are in form of aggregation, and the interaction between aggregated $A \beta$ is minimum as shown by our ITC data (almost no binding enthalpy were observed, data not shown). Also, from the SPR sensorgram, the lowest Ka value for $A \beta$ with DPPC/GM1/cholesterol $(5: 3: 2)$ is caused by the higher $k_{\mathrm{d}}$ value (dissociation constant) of the binding system. Therefore, the specific binding between the GM1 with $A \beta$ play less profound role with $\mathrm{A} \beta$ aggregates.

\subsubsection{Binding enthalpy measurements}

Fig. 6 shows the heat of reaction of interaction between fresh $A \beta$ (1-40) and various liposomes measured by ITC. It can be seen that the interaction between $A \beta$ and liposomes is endothermic in nature. Liposomes, such as DPPC and DPPC/20\% cholesterol had relatively larger values of enthalpy, while others showed $A \beta$ has lower enthalpy values of the magnitude of $1 \mathrm{~kJ} / \mathrm{mol}$. It was found that smaller enthalpy values were obtained for liposomes that have a negative charge. This suggests that the $A \beta$-liposome interaction is predominantly electrostatic-driven due to the large extent of adverse contribution from the electrostatic force, thus, leading to a smaller heat reaction. However, for liposomes with uncharged lipids, the driving force of $A \beta$-liposome interaction is hydrophobic in nature.

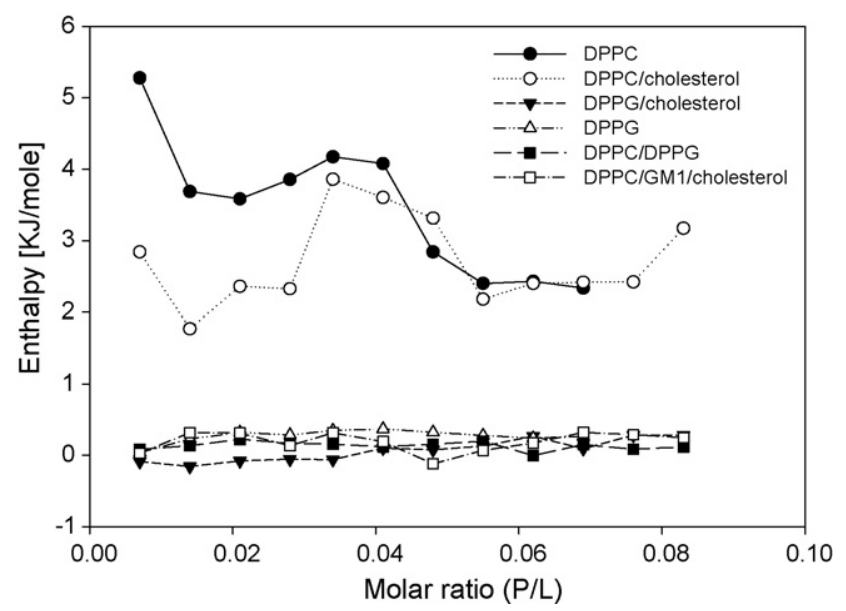

Fig. 6. The reaction heat of fresh $\mathrm{A} \beta$ (1-40) with various liposome. The liposomes include DPPC, DPPC/20\% cholesterol, DPPG, DPPG/20\% cholesterol, DPPC/DPPG (3:1 molar ratio) and DPPC/GM1/cholesterol (5:3:2 molar ratio). The peptide/lipid molar ratio of the range: $0.007-0.083$. 


\section{Conclusions}

\subsection{A $\beta$ structure}

From the initial stage to the completion of 1-day incubation, $\mathrm{A} \beta$ showed a structural transition from a particulate matter to fibrillar. This was observed from the results obtained from AFM, $\mathrm{CD}$ and ThT fluorescence measurements.

\subsection{Kinetics}

The interaction between fresh $\mathrm{A} \beta$ and liposome was examined. Electrostatic force was found to be significant for $A \beta$ and liposome prior to incubation. However, after incubation for a single day, hydrophobicity was the driving force behind $\mathrm{A} \beta$ and liposome interaction. Hence, fresh and incubated $A \beta$, though only for a day, had its own distinctive interacting mechanism.

\subsection{Binding enthalpy}

Data from heat of reaction indicated that both electrostatic and hydrophobic force was responsible for the interaction between fresh $A \beta$ and liposome. The negative electrical surface charge of liposome has been the essential driving force responsible for $A \beta$-liposome interaction. In contrast with liposome without surface charge, the dominant driving force was hydrophobicity. Therefore, surface change and polarity, determined by the composition of liposomes, resulted in different binding mechanisms between fresh $\mathrm{A} \beta$ and liposomes.

\section{Appendix A}

The binding mechanism was described by Langmuir binding model. $\mathrm{P}$ and $\mathrm{L}$ denote the peptide and lipid, respectively.

$\mathrm{P}+\underset{k_{\mathrm{off}}}{\mathrm{L}} \stackrel{k_{\mathrm{gn}}}{\Leftrightarrow} \mathrm{PL}$

The dissociation rate constant $k_{\text {off }}$ was derived using the following equation:

$R_{t}=R_{t_{\mathrm{o}}} \mathrm{e}^{-k_{\text {off }}\left(t-t_{\mathrm{o}}\right)}$

where $R_{t}$ is the response at time $t$ and $R_{t_{\mathrm{o}}}$ is the initial response. The association rate constant $\mathrm{k}_{\mathrm{on}}$ was obtained via the equation below:

$R_{t}=\frac{k_{\mathrm{on}} C R_{\max }\left(1-\mathrm{e}^{-\left(k_{\mathrm{on}} c+k_{\mathrm{off}}\right) t}\right)}{k_{\mathrm{on}} C+k_{\mathrm{off}}}$

where $R_{\max }$ is the maximum response and $C$ is the peptide concentration in the solution.

Affinities were calculated from the rate constants

$K_{\mathrm{A}}=\frac{k_{\mathrm{on}}}{k_{\mathrm{off}}}$

$K_{\mathrm{A}}$ is defined as the affinity constant.

\section{References}

[1] D. Foguel, J.L. Silva, Biochemistry 43 (2004) 11362-11370

[2] M. Wakabayashi, T. Okada, Y. Kozutsumi, K. Matsuzaki, Biochem. Biophys. Res. Commun. 328 (2005) 1019-1023.

[3] J.D. Green, L. Kreplak, C. Goldsbury, X. Li Blatter, M. Stolz, G.S. Cooper, A. Seelig, J. Kistler, U. Aebi, J. Mol. Biol. 342 (2004) 877-887.

[4] W.G. Wood, G.P. Eckert, U. Igbavboa, W.E. Muller, Biochim. Biophys. Acta 1610 (2003) 281-290.

[5] X. Yanming, K. Higuchi, Mech. Ageing Dev. 123 (2002) 1625-1636.

[6] O. Gursky, S. Aleshkov, Biochim. Biophys. Acta 1476 (2000) 93-102.

[7] R. Sabate, M. Gallardo, J. Estelrich, Int. J. Biol. Macromol. 35 (2005) 9-13.

[8] C.L. Shen, R.M. Murphy, Biophys. J. 69 (1995) 640-651.

[9] S.J. Wood, B. Maleeff, T. Hart, R. Wetzel, J. Mol. Biol. 256 (1996) 870-877.

[10] T. Ban, M. Hoshino, S. Takahashi, D. Hamada, K. Hasegawa, H. Naiki, Y. Goto, J. Mol. Biol. 344 (2004) 757-767.

[11] M. Bokvist, F. Lindstrom, A. Watts, G. Grobner, J. Mol. Biol. 335 (2004) 1039-1049.

[12] L.P. Choo-Smith, W.K. Surewicz, FEBS Lett. 402 (1997) 95-98.

[13] Y. Tashima, R. Oe, S. Lee, G. Sugihara, E.J. Chamers, M. Takahashi, T. Yamada, J. Biol. Chem. 279 (2004) 17587-17595.

[14] C.S. Goldsbury, S. Wirtz, S.A. Muller, S. Sunderji, P. Wicki, U. Aebi, P. Frey, J. Struct. Biol. 130 (2000) 217-231.

[15] E. Terzi, G. Holzemann, J. Seelig, J. Mol. Biol. 252 (1995) 633-642.

[16] J.J. Kremer, R.M. Murphy, J. Biochem. Biophys. Methods 57 (2003) 159-169.

[17] A. Kakio, Y. Yano, D. Takai, Y. Kuroda, O. Matsumoto, Y. Kozutsumi, K. Matsuzaki, J. Peptide Sci. 10 (2004) 612-621.

[18] T. Ariga, K. Kobayashi, A. Hasegawa, M. Kiso, H. Ishida, T. Miyatake, Arch. Biochem. Biophys. 388 (2001) 225-230.

[19] J.J. Kremer, M.M. Pallitto, D.J. Sklansky, R.M. Murphy, Biochemistry 39 (2000) 10309-10318.

[20] J. Lahiri, P. Kalal, A.G. Frutos, S.J. Jonas, R. Schaeffler, Langmuir 16 (2000) 7805-7810.

[21] H. LeVine, Protein Sci. 2 (1993) 404-410.

[22] L.-Y. Chen, J.-J. Lin, M.-S. Lin, S.-M. Chiu, Y. Chang, W.-Y. Chen, Colloids Surf. A 296 (2007) 86-91.

[23] W.P. Hu, S.J. Chen, K.T. Huang, J.H. Hsu, W.Y. Chen, G.L. Chang, K.A. Lai, Biosens. Bioelectron. 19 (2004) 1465-1471.

[24] T. Valdes-Gonzalez, J. Inagawa, T. Ido, Peptides 22 (2001) 1099-1106. 